\title{
Comparison of del Nido Cardioplegia and Blood Cardioplegia in Aortic Root Surgery
}

\author{
Yusuf Kuserli, Saygin Turkyilmaz, Gulsum Turkyilmaz, Ali Aycan Kavala \\ Department of Cardiovascular Surgery, Bakirkoy Dr. Sadi Konuk Training and Research Hospital, Istanbul, Turkey
}

\section{ABSTRACT}

Aim: To compare del Nido cardioplegia (DNC) with conventional blood cardioplegia (BC) in aortic root surgery.

Methods: Subjects who underwent aortic root surgery during a 3-year period were included. A DNC group was compared with a matched BC group.

Results: A total of 72 subjects were included, 36 who underwent DNC compared with 36 propensity-matched subjects who underwent BC. Fifty-one (70.8\%) were male, and $21(29.2 \%)$ were female, with a mean age of $66.19 \pm 7.02$ years (range 51 to 81 ). No significant differences in baseline characteristics, preoperative echocardiogram parameters, or intraoperative parameters were found between the groups. For DNC versus BC, cardiopulmonary bypass time, aortic clamp time, cardioplegia volume (all $P=.001$ ), and defibrillation $(P=.007)$ were significantly lower. For postoperative biochemical parameters, creatinine levels at hour 24, potassium levels at hours 1 and 24, and glucose levels at hours 6 and 24 did not differ between the groups $(P>.05)$. Creatine kinase-MB and troponin T levels at hours 1 and 24 were significantly lower in DNC versus BC (all $P=.001$ ). Hematocrit levels at hours 6 and 24 were significantly higher in DNC $(P=.001)$. The groups did not differ in terms of postoperative inotropic support, postoperative complications, intubation period, or duration of intensive care unit stay $(P>.05)$. Although the need for thrombocyte transfusion did not differ between groups $(P>.05)$, DNC resulted in less use of erythrocyte and fresh frozen plasma transfusions (both $P=.001$ ). Postoperative ejection fraction was significantly better in the DNC group than in the BC group $(P=.006)$.

Conclusion: The results indicate better intraoperative parameters and better ejection fraction rates with DNC than with BC. DNC is an effective and safe alternative to blood cardioplegia for aortic root surgery.

\section{INTRODUCTION}

Myocardial protection is an important area of research for cardiac surgery [Gorgy 2019]. Different cardioplegia solutions have been used since the 1950s for myocardial protection

Received Fanuary 4, 2020; accepted March 10, 2020.

Correspondence: Saygin Turkyilmaz, MD; 90-505-255-79-93; (e-mail: sygnty@hotmail.com).
[Nardi 2018]. The ideal cardioplegia solution needs to be safe, effective, and easy to administer. If possible, it needs to reduce the operative time and cost of the operation [Rosenbloom 2018; Ota 2016]. Cardioplegia solutions are crystalloid or bloodbased solutions with various chemical compounds. The delivery method (antegrade-retrograde), temperature (warm or cold), and timing (intermittent or continuous) change according to the formulations [Vistarini 2017]. Conventional blood cardioplegia has been widely used for myocardial protection for decades.

The use of cardioplegia solution varies according to geographical area and shows a wide variation across countries. In a global survey that evaluated cardioplegia practices, Ali et al [2018] reported that the most commonly used cardioplegia solutions are St. Thomas' hospital solution (45.8\%), Bretschneider solution (14.0\%), and the University of Wisconsin solution (14.8\%). However, Del Nido cardioplegia (DNC) was used in only $4.1 \%$ of the respondents who were from North America [Ali 2018].

DNC offers single-dose, antegrade cardioplegia that is easy to administer and has a long redosing interval [Kim 2014]. DNC has been used for pediatric heart surgery for 20 years with demonstrated safety and efficacy. Although promising results have been obtained in adult cardiac surgery, a standard protocol and data on its use for multiple/complex cardiac surgery are lacking [Kim 2018; Valooran 2016]. In the available literature, we could not find a study that evaluated DNC in aortic root surgery. The aim of the current study was to compare del Nido cardioplegia with conventional blood cardioplegia in aortic root surgery.

\section{METHODS}

A retrospective study was performed at Bakırköy Dr. Sadi Konuk Teaching and Research Hospital between May 2015 and May 2018, a 3-year period. The study protocol was approved by the local ethics board.

Subjects of all ages who underwent aortic root surgery were included in the study. Subjects with

- Pediatric age ( $<18$ years);

- Previous cardiac surgery (open);

- Concomitant cardiac surgery;

- Aortic dissection; or

- Infective endocarditis

were excluded.

For baseline characteristics, subjects' age, sex, body mass index (BMI), New York Heart Association (NHYA) 
Table 1. Cardioplegia solution compositions

Cardioplegia solution compositions

Del Nido cardioplegia solution: Plasma-Lyte A (1:4) composition (total volume $1060 \mathrm{~mL}$ )
Blood cardioplegia solution: Ringer lactate (1:4) composition (total volume $550 \mathrm{~mL}$ )

$\begin{array}{lc}\text { Mannitol } 20 \% & 16.3 \mathrm{~mL}(3.26 \mathrm{~g}) \\ \text { Potassium chloride, } 2 \mathrm{mEq} / \mathrm{mL} & 13 \mathrm{~mL}(26 \mathrm{mEq}) \\ \text { Magnesium sulphate } 50 \% & 4 \mathrm{~mL}(2 \mathrm{~g}) \\ \text { Lidocaine } 1 \% & 13 \mathrm{~mL}(130 \mathrm{mg}) \\ \text { Sodium bicarbonate } 8.4 \% & 13 \mathrm{~mL}(13 \mathrm{mEq})\end{array}$

functional status, cardiac comorbidities (hypertension, dyslipidemia, cardiac rhythm, aortic insufficiency, aortic stenosis, or bicuspid aorta) and noncardiac comorbidities (tobacco use, alcohol consumption, diabetes mellitus, chronic obstructive pulmonary disease, chronic renal insufficiency, preoperative transient ischemic attack [TIA], or stroke) were recorded. Preoperative echocardiogram parameters (ejection fraction $[\mathrm{EF}]$, valvular area, and valve gradient) were also noted.

\section{Surgical Procedure}

A pericardiotomy was performed following median sternotomy under general anesthesia. The pericardium was suspended with silk sutures. According to the surgical technique, arterial cannulation was performed from the ascending aorta, femoral artery, and axillary artery. Venous cannulation was performed in all subjects using 2 -stage venous cannulas from the right atrium. In all subjects, the vent cannula was placed into the right superior pulmonary vein. Under cardiopulmonary bypass $(\mathrm{CPB})$, when the temperature reached $32^{\circ} \mathrm{C}$, an aortic cross clamp was placed. The initial cardioplegia dose for both DNC and blood cardioplegia (BC) was $1000 \mathrm{~mL}$. Aortotomy was performed. Only antegrade cardioplegia was administered from the coronary ostium.

According to the condition of the aortic valve and coronary ostia, valve sparing surgery (David operation) or total aortic root replacement (Button Bentall or Cabrol operation) was performed. Coronary ostia were separated and released from the aorta in a button-shaped fashion. The size of the valve and graft was determined according to the aortic annulus. According to the preferred surgical technique, aortic root replacement was performed with a valve conduit or Dacron graft. The ostia were opened to the appropriate positions on the graft, and the left and right coronary buttons were anastomosed to the graft with $6 / 0$ Prolene. In the subjects who underwent the Cabrol operation, a 10-mm Dacron graft was used for reimplantation of the coronary ostium. Distal anastomosis of the valve conduit or flat Dacron graft was made with 4/0 Prolene with the ascending distal aorta.

The aortic cross clamp was removed, and under appropriate hemodynamic conditions, CPB was ended, and decannulation was performed. Drains were placed for bleeding control. The sternum was then sutured with wire and closed.

$\begin{array}{cc}\mathrm{Na}^{+} & 110 \mathrm{mmol} / \mathrm{L} \\ \mathrm{K}^{+} & 16 \mathrm{mmol} / \mathrm{L} \\ \mathrm{Mg}^{2+} & 32 \mathrm{mmol} / \mathrm{L} \\ \mathrm{Ca}^{2+} & 2.4 \mathrm{mmol} / \mathrm{L} \\ \text { Bicarbonate } & 10 \mathrm{mmol} / \mathrm{L} \\ \mathrm{Cl}^{-} & 160 \mathrm{mmol} / \mathrm{L}\end{array}$

The fascia and subcutaneous tissue were closed with $2 / 0$ Vicryl sutures, and the skin was closed with $3 / 0$ atraumatic Vicryl subcuticular continuous sutures. DNC redosing was performed every 60 minutes, and $\mathrm{BC}$ redosing was performed every 20 minutes with half of the initial dose. The temperature of cardioplegia delivery for DNC and $\mathrm{BC}$ was between $4^{\circ} \mathrm{C}$ and $8^{\circ} \mathrm{C}$. The compositions of the del Nido and blood cardioplegia solutions that were used during the surgery are presented in Table 1.

For intraoperative parameters, operation type (Bentall, David, or Cabrol), CPB, aortic clamp time, hypothermic circulatory arrest (HCA) time, cardioplegia volume, minimum heat, clamped aortic anastomosis, arterial cannulation area (ascending aorta, femoral artery, or axillary artery), and defibrillation were noted.

For postoperative biochemical parameters, creatine kinase (CK)-MB (at hours 1 and 24), troponin T (at hours 1 and 24), hematocrit (at hours 6 and 24) creatinine (at hour 24), potassium (at hours 1 and 24) and glucose levels (at hours 6 and 24) were noted. In the postoperative period, red cell transfusion was given for hematocrit values $<24$.

Postoperative complications, including low CO, myocardial infarction, acute renal insufficiency, atrial fibrillation/ other arrhythmias, respiratory insufficiency, TIA/stroke, transient pacemaker need, reoperation due to bleeding, infection, and hospital mortality, were noted. The presence of inotropic support and the support type (norepinephrine or other) in the first 24 hours was noted. The intubation period and duration of intensive care stay were also noted. All subjects underwent an echocardiographic assessment for EF before discharge from the hospital.

\section{Propensity Matching}

Multivariate logistic regression analysis was performed to calculate propensity scores for the 2 groups using the following covariates: age, sex, chronic obstructive pulmonary disease, hypertension, diabetes mellitus, cerebrovascular accident, chronic renal insufficiency (defined as serum creatinine $>2 \mathrm{mg} / \mathrm{dL}$ ), NYHA classification, chronic heart failure (defined as EF 30\%), myocardial infarction, and shock. The Greedy 5-to-1 digit matching technique was then used to select patients undergoing aortic root surgery with del Nido 
Table 2. Subjects' baseline characteristics according to cardioplegia type $(\mathrm{N}=72)$

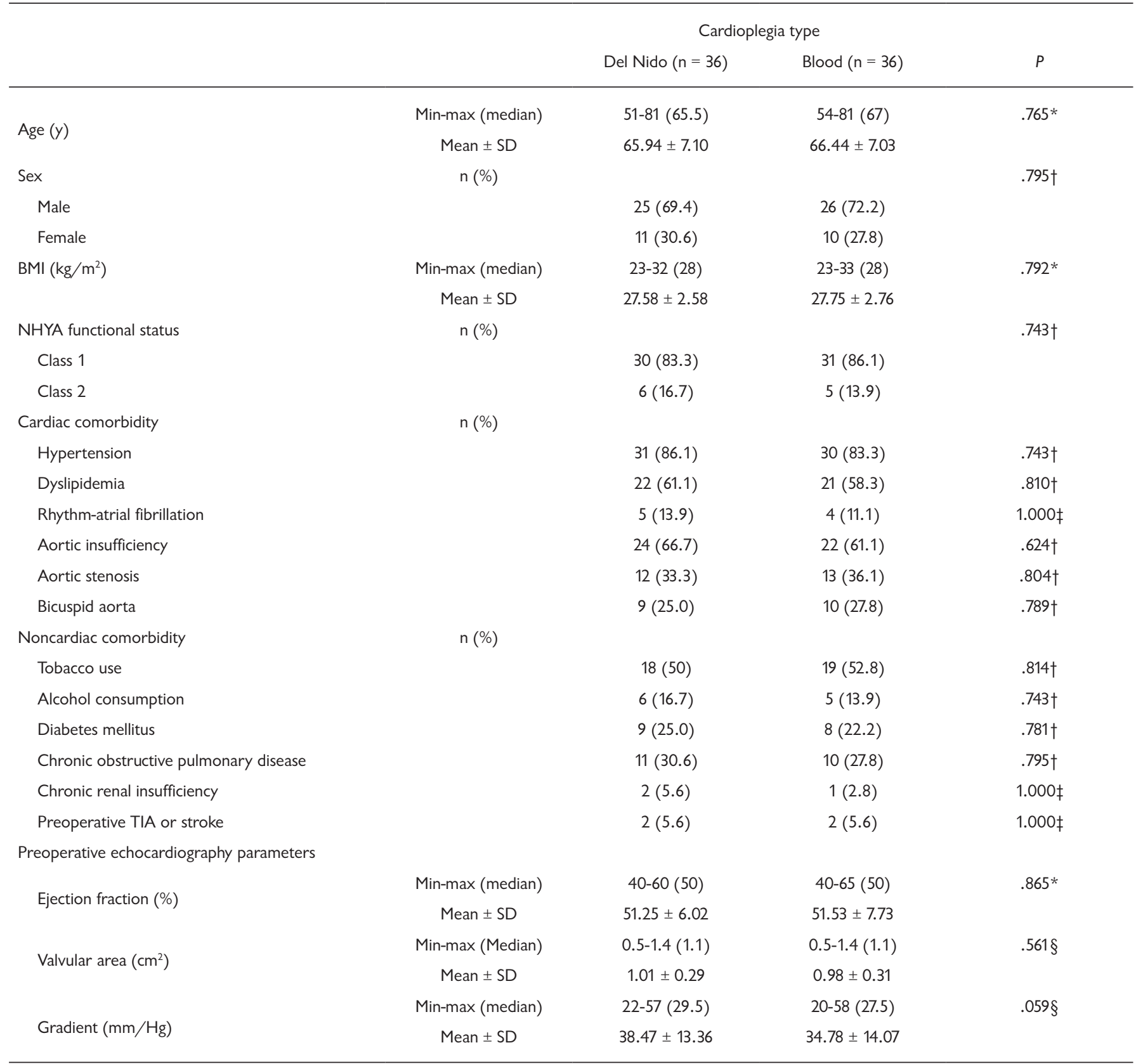

*Student’s $t$ test; $\nmid$ Pearson $\chi^{2}$ test; $\ddagger$ Fisher’s exact test; $\S$ Mann-Whitney $U$ test.

cardioplegia solution as counterparts to those with conventional blood cardioplegia by choosing the patient with the nearest propensity score. The C-statistic for this propensity model was 0.82 .

\section{Statistical Analysis}

Number Cruncher Statistical System (NCSS) 2007 (Kaysville, UT) was used for the statistical analysis. Descriptive statistical methods (minimum, maximum, standard deviation
(SD), mean, median, frequency, and percentage) were used. Quantitative data were evaluated with the Shapiro-Wilk test and graphical evaluations. Quantitative data with a normal distribution were compared with Student's $t$ test, and quantitative data without a normal distribution were compared using the Mann-Whitney $\mathrm{U}$ test. Comparisons in groups for data with a normal distribution were performed with pairedsample $t$ tests, and comparisons in groups for data without a normal distribution were performed with the Wilcoxon sign 


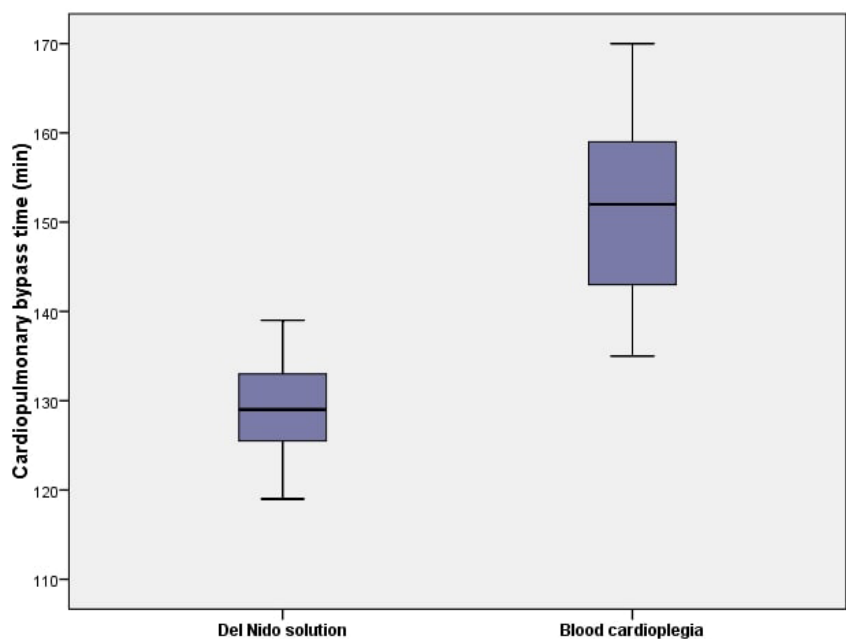

Figure 1. Comparison of cardiopulmonary bypass time (minutes) between del Nido cardioplegia and blood cardioplegia.

test. For qualitative data, Pearson's $\chi^{2}$ test, Fisher's exact test, and Fisher-Freeman-Halton test were used. For the analysis, a $P$ value $<.05$ was considered significant.

\section{RESULTS}

In total, 72 subjects were evaluated. Both the DNC and BC groups consisted of 36 subjects. Fifty-one subjects $(70.8 \%)$ were male, and $21(29.2 \%)$ were female. The mean age of the subjects was $66.19 \pm 7.02$ years (range 51 to 81 ). There were no significant differences in baseline characteristics in terms of age, sex, BMI, NHYA functional status, cardiac comorbidities, or noncardiac comorbidities. Preoperative echocardiogram parameters (EF, valvular area, and valve gradient) were also not significantly different ( $P>.05$ for all comparisons). The baseline characteristics of the groups are presented in Table 2.

For intraoperative parameters, the groups did not differ in terms of operation type (Bentall, David, or Cabrol), HCA time, minimum heat, clamped aortic anastomosis, or arterial cannulation (ascending aorta, femoral artery, or axillary artery) ( $P=1.000, .198, .727$, and .427 , respectively). Comparing DNC with BC, CPB time $(129.39 \pm 5.72$ and 151.94 $\pm 10.12 \mathrm{~min}$, respectively, $P=.001$ ) (Figure 1 ), aortic clamp time $(96.75 \pm 5.24$ and $116.42 \pm 9.27 \mathrm{~min}, P=.001)($ Figure 1$)$, cardioplegia volume $(1901.39 \pm 179.08$ and $3954.86 \pm 490.66$ $\mathrm{mL}, P=.001)$, and defibrillation $(\mathrm{n}=8,22.2 \%$, and $\mathrm{n}=19$ $52.8 \%, P=.007)$ were significantly lower in the DNC group than the BC group. Intraoperative data according to cardioplegia type are presented in Table 3.

For postoperative biochemical parameters, creatinine levels at hour 24, potassium levels (at hours 1 and 24), and glucose levels (at hours 6 and 24) did not differ between the groups ( $P>.05$ for all comparisons) (Table 4). CK-MB levels at hour $1(5.88 \pm 2.48 \mathrm{DNC}$ and $8.02 \pm 2.00 \mathrm{ng} / \mathrm{mL} \mathrm{BC}$, $P=.001)$ and hour $24(13.40 \pm 4.38$ and $16.96 \pm 3.57 \mathrm{ng} / \mathrm{mL}$,

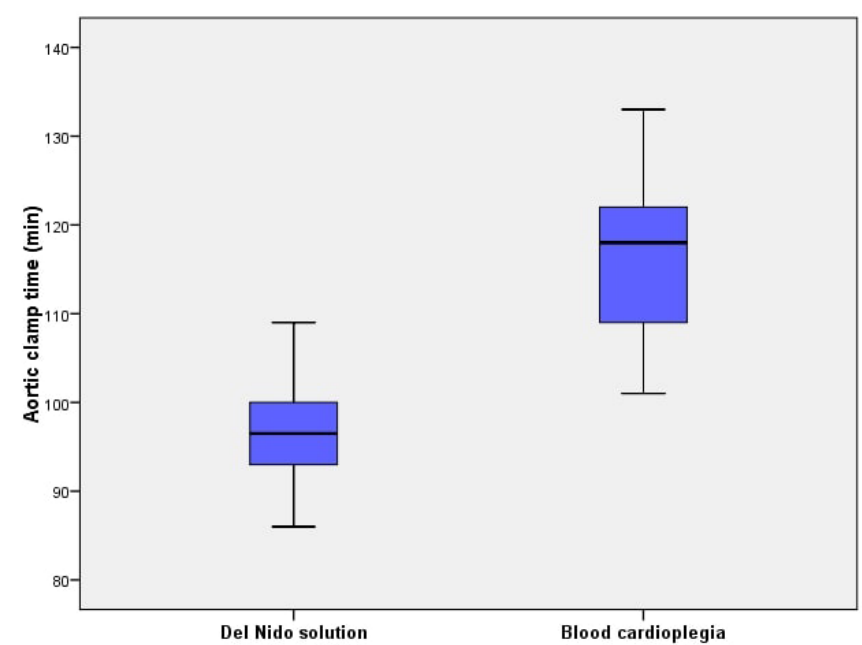

Figure 2. Comparison of aortic clamp time (minutes) between del Nido cardioplegia and blood cardioplegia.

$P=.001)$ and troponin T levels at hour $1(0.62 \pm 0.33$ and 0.99 $\pm 0.40 \mathrm{ng} / \mathrm{mL}, P=.001)$ and hour $24(0.34 \pm 0.22 \mathrm{ng} / \mathrm{mL}$ and $0.73 \pm 0.35 \mathrm{ng} / \mathrm{mL}, P=.001)$ were significantly lower in the DNC group than the $\mathrm{BC}$ group. Hematocrit levels at hour 6 $(33.06 \pm 1.85$ and $29.31 \pm 1.33 \mathrm{ng} / \mathrm{mL}, P=.001)$ and hour 24 $(33.08 \pm 1.27$ and $30.78 \pm 0.76 \mathrm{ng} / \mathrm{mL}, P=.001)$ were significantly higher in the DNC group than the BC group.

The groups did not differ in terms of postoperative complications, postoperative inotropic support, intubation period, or duration of intensive care unit stay $(P>.05$ for all comparisons) (Table 5).

Although need for thrombocyte transfusion did not differ between the groups $(P>.05)$, DNC resulted in less use of erythrocyte $(0.47 \pm 0.74$ and $1.75 \pm 0.97 \mathrm{U}, P=.001)$ and fresh frozen plasma $(0.33 \pm 0.63$ and $1.14 \pm 0.87 \mathrm{U}, P=.001)$ transfusions. Postoperative EF was significantly better in the DNC group than in the BC group $(53.75 \% \pm 6.14 \%$ and $50.00 \% \pm$ $5.07 \%, P=.006)$.

\section{DISCUSSION}

We report a comparative study that evaluated the efficacy of DNC in aortic root surgery. The results indicate that compared with the use of $\mathrm{BC}$, the use of DNC results in shorter aortic clamp and $\mathrm{CPB}$ times. DNC required a lower cardioplegia volume, and defibrillation events occurred less frequently. The biochemical parameters of CK-MB and troponin were significantly lower, and hematocrit levels were significantly higher, in the DNC group than the BC group. Compared with BC, DNC also resulted in fewer erythrocyte and fresh frozen plasma transfusions.

Myocardial protection is an essential component of complex cardiac surgery [Gorgy 2019]. Different formulations have existed since the 1950s. Initial cold crystalloid cardioplegia was followed by warm blood cardioplegia; however, most of the studies published in the 1980s and 1990s did not report 
Table 3. Intraoperative data according to cardioplegia type

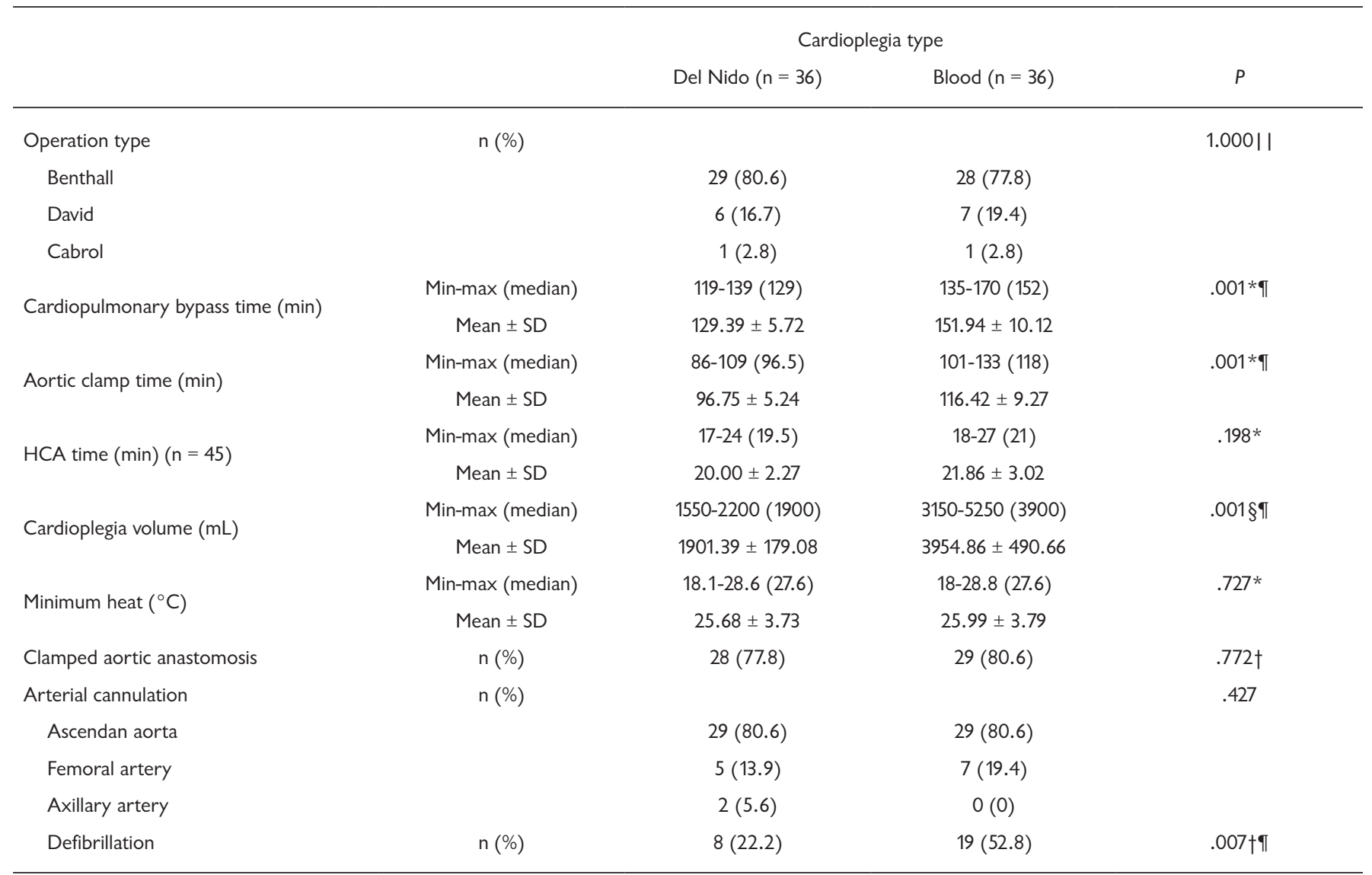

*Student’s $t$ test; $\nmid$ Pearson $\chi^{2}$ test; $¥$ Fisher’s exact test; §Mann-Whitney $U$ test; | |Fisher-Freeman-Halton test; $\llbracket P<.01$.

significant differences according to type (crystalloid/blood), temperature (cold/tepid/warm), or administration route (antegrade/retrograde) between cardioplegia types [Nardi 2018; Kim 2018]. More recently, DNC was introduced for neonatal heart surgery. Recent use of DNC has shown that it offers better myocardial protection; however, data are lacking, especially in areas such as complex cardiac surgery [Gorgy 2019]. The use of DNC in adult cardiac surgery started with minimally invasive or less complex procedures [Ad 2018].

Every effort should be taken by surgeons to shorten the operation time as well as the aortic cross clamp and CPB times to improve surgical outcomes. In 5006 subjects, Salis et al [2008] reported that CPB time was an independent risk factor for postoperative complications (including renal, pulmonary, and neurologic complications) and postoperative mortality. In 27,215 subjects, Doenst et al [2008] reported that CPB time was an independent predictive factor for mortality. Previous studies supported these effects in different cardiac procedures [Vistarini 2017]. Ota et al [2016] reported the outcomes of DNC in 54 pair-matched subjects. The use of DNC resulted in a shorter mean CPB time $(71 \pm 16 \mathrm{~min}$ for DNC and 84 $\pm 28 \mathrm{~min}$ for $\mathrm{BC})$ and mean aortic cross-clamp time (52 \pm $14 \mathrm{~min}$ for DNC and $60 \pm 16 \mathrm{~min}$ for $\mathrm{BC}$ ). Postoperative inotropic support and postoperative complications were similar. In 46 subjects (25 DNC and $21 \mathrm{BC}$ ), Vistarini et al [2017] reported a smaller cardioplegia volume, less ventricular fibrillation, a lower postoperative CK-MB level, and less need for the postoperative use of intravenous insulin in the DNC group. The CPB time, aortic clamp time, intubation period, mean hospital stay, and complication rate were similar. Sorabella et al [2014] compared DNC $(\mathrm{n}=52)$ with BC $(\mathrm{n}=61)$ in 113 subjects who underwent aortic valve repair. Both groups demonstrated similar cross-clamp times, bypass times, postoperative complication rates, and types of patients. The DNC group demonstrated lower total cardioplegia volume and cardioplegia dose.

Among published studies, only 1 randomized controlled study exists in the literature. Ad et al [2018] conducted the study on first-time coronary artery bypass grafting (CABG), valve, or $\mathrm{CABG} /$ valve surgery subjects. In 89 subjects (DNC, $\mathrm{n}=48 ; \mathrm{BC}, \mathrm{n}=41$ ), the $\mathrm{CPB}$ time (97 versus 103 minutes; $P=.288$ ) and cross-clamp time (70 versus 83 minutes; $P=.018$ ) were not significantly different between the groups. Although the nonsignificant return to spontaneous rhythm was higher, the inotropic support requirement and troponin levels were lower in the DNC group. The authors suggested that the use 
Table 4. Postoperative biochemical parameters according to cardioplegia type

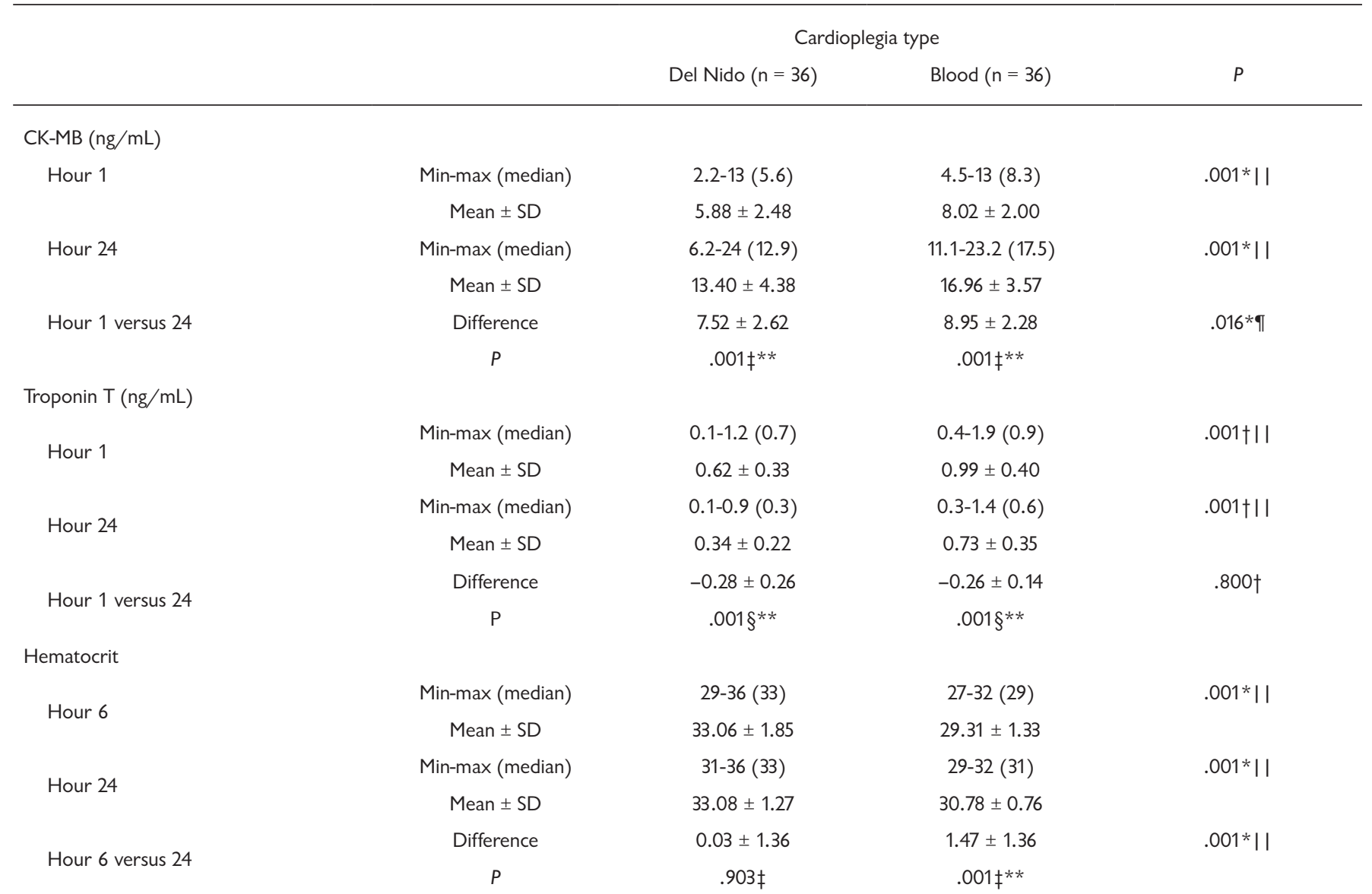

of troponin levels should continue in future studies, as it is a sign of superior myocardial protection with DNC.

Data on DNC started to be retrieved from pooled metaanalyses. A recent meta-analysis that included 9 studies (4 that were conducted for adult valve surgery, 3 for CABG surgery, and 2 for valve/CABG or a combination of valve/ CABG surgery) compared DNC with conventional cardioplegia [Li 2018]. In 1501 subjects, the results indicated that $\mathrm{DNC}$ reduced the $\mathrm{CPB}$ and cross-clamp times. The cardioplegia volume was lower, and the blood glucose level was reduced with DNC. DNC shortened the intensive care unit stay, but myocardial enzyme levels, postoperative inotropic support need, lengths of hospital stay, and mortality rates were not significantly different between the DNC and $\mathrm{BC}$ groups.

The most recent meta-analyses between 1996 and 2017 include 7 adjusted $(\mathrm{n}=1104)$ and 5 unadjusted $(\mathrm{n}=717)$ observational studies and 1 randomized controlled trial $(\mathrm{n}=$ 89) [An 2019]. Among these studies, 1 study evaluated aortic/ mitral valve surgery, 3 evaluated isolated CABG surgery, 4 evaluated aortic valve surgery, and 5 evaluated both valve and CABG surgeries. DNC reduced the cardioplegia volume by $1.1 \mathrm{~L}$ and decreased the aortic cross-clamp and $\mathrm{CPB}$ times by 8 minutes. DNC also decreased troponin release $(0.3 \mathrm{ng} / \mathrm{mL})$.
Other parameters, such as complications, transfusion needs, reoperation rates, postoperative EF, and hospital rates, were similar between the groups.

We could not find a specific study regarding aortic root surgery. However, in a mixed study, Kim et al [2018] evaluated 149 procedures that used DNC. Thirteen of 149 procedures were aortic root procedures, but the results were given for the total group. Overall, the DNC group demonstrated lower peak troponin I levels and shorter aortic clamping times. Early mortality rates, low cardiac output, and neurological events were similar. The number of postoperative transfusions and the need for fluid supplementation were significantly lower in the DNC group than the BC group.

DNC offers some advantages with its own ingredients. PlasmaLyte, which is the balanced base solution, is iso-osmolar (osmolarity $294 \mathrm{mmol} / \mathrm{L}$ ) and has a physiological $\mathrm{pH}$. Its physiological nature does not affect red blood cell physiology [Nardi 2018]. Lidocaine is an important ingredient that allows membrane stabilization and decreases excitability. By blocking sodium channels, lidocaine blocks spontaneous myocardial contractions and reduces the release of troponin I. This is especially beneficial for reducing arrhythmias, which may complicate cardiac surgery. In combination with magnesium (as a calcium channel blocker), lidocaine reduces 
Table 4. Postoperative biochemical parameters according to cardioplegia type [cont.]

\begin{tabular}{|c|c|c|c|c|}
\hline \multirow{2}{*}{ Preoperative } & Min-max (median) & $0.6-6.8(0.9)$ & $0.4-6.8(0.8)$ & $.055 \dagger$ \\
\hline & Mean \pm SD & $1.18 \pm 1.16$ & $0.97 \pm 1.03$ & \\
\hline Hour 24 & Mean \pm SD & $1.22 \pm 1.26$ & $1.38 \pm 1.31$ & \\
\hline \multirow{2}{*}{ Preoperative versus hour 24} & Difference & $0.04 \pm 0.19$ & $0.41 \pm 1.06$ & $.001 \dagger \mid l$ \\
\hline & $P$ & $.371 \S$ & $.001 \S^{* *}$ & \\
\hline \multicolumn{5}{|l|}{ Potassium } \\
\hline Hour 1 & Min-max (median) & $3.4-4.2(3.9)$ & $3.5-4.2(3.9)$ & $.736 *$ \\
\hline \multirow{2}{*}{ Hour 1 versus 24} & Difference & $-0.07 \pm 0.14$ & $0.01 \pm 0.16$ & $.013 * \mathbb{\pi}$ \\
\hline & $P$ & $.002 \ddagger^{* *}$ & $.603 \ddagger$ & \\
\hline \multicolumn{5}{|l|}{ Glucose } \\
\hline \multirow{2}{*}{ Hour 6} & Min-max (median) & $80-281(91.5)$ & $82-287(90.5)$ & $.901 \dagger$ \\
\hline & Mean \pm SD & $119.28 \pm 55.87$ & $122.42 \pm 65.53$ & \\
\hline \multirow{2}{*}{ Hour 24} & Min-max (median) & $81-261(89)$ & $80-291(89)$ & $.888 \dagger$ \\
\hline & Mean \pm SD & $118.28 \pm 56.79$ & $119.89 \pm 62.57$ & \\
\hline \multirow[b]{2}{*}{ Hour 6 versus 24} & Difference & $-1.00 \pm 9.04$ & $-2.53 \pm 10.64$ & $.689 \dagger$ \\
\hline & $P$ & $.771 \S$ & $.369 \S$ & \\
\hline
\end{tabular}

*Student’s $t$ test; $\nmid$ Mann-Whitney $U$ test; $\ddagger$ Paired samples $t$ test; $\S$ Wilcoxon signed ranks test; $\| P<.01 ; \llbracket P<.05$.

myocardial edema, which offers an advantage for better myocardial protection. Sodium gluconate has an alkalizing effect that is helpful for balancing myocardial $\mathrm{pH}$. High-potassium ingredients also allow better diastolic arrest and a flaccid myocardium [Kim 2014]. Del Nido solution also has specific advantages, as it does not contain calcium or glucose, which may have specific negative effects on surgery [Kim 2018]. Myocardial injury during cardiac arrest is strongly related to the accelerated accumulation of intracellular calcium. The above properties all offer a decreased accumulation of intracellular calcium. Cold delivery of del Nido solution decreases myocardial oxygen consumption. DNC provides a myocardial cooling of $<15^{\circ} \mathrm{C}$, and every $10^{\circ} \mathrm{C}$ decrease in myocardial temperature results in a $50 \%$ decrease in oxygen consumption [Vistarini 2017].

In addition to $\mathrm{CPB}$ time and cross-clamp time, $\mathrm{CK}-\mathrm{MB}$ and troponin levels are the objective measurements linked directly to the quality of myocardial protection. CK-MB and troponin levels are also independent factors related to cardiac surgery mortality [Li 2018]. CK-MB levels at hours 1 and 24 and troponin $\mathrm{T}$ levels at hours 1 and 24 were significantly lower in the DNC group than in the BC group. Lower levels of troponin $\mathrm{T}$ and $\mathrm{CK}-\mathrm{MB}$ were both related to $\mathrm{DNC}$ use and lower cross-clamp and CPB times.

Data from the literature have demonstrated that DNC has a benefit in terms of quickly resuming spontaneous regular cardiac rhythm [Mukdad 2018]. DNC resulted in less of a requirement for defibrillation to obtain a normal rhythm compared with BC (22\% for DNC and $52.8 \%$ for BC) in our study. Although there was no correlation between defibrillation and myocardial protection, a high percentage of spontaneous return from arrest to a sinus rhythm and rare defibrillation requirements are signs of good myocardial protection [Vistarini 2017].

DNC has an advantage in terms of blood glucose levels. In a multicenter study of 1118 matched subjects from 17 cardiac centers, reported that subjects who received $\mathrm{BC}$ required more intraoperative insulin use compared with subjects who received DNC $(65.7 \%$ versus $56.2 \% ; P<$ $.001)$. The use of DNC resulted in a $5.7 \mathrm{mg} / \mathrm{dL}$ decrease in blood glucose levels compared with the use of BC. DNC and $\mathrm{BC}$ did not differ in terms of glucose levels in our study. Ota et al [2016] speculated that the effect of cardioplegia solution on hematocrit levels is minimized by 
Table 5. Transfusion and other postoperative parameters according to cardioplegia type*

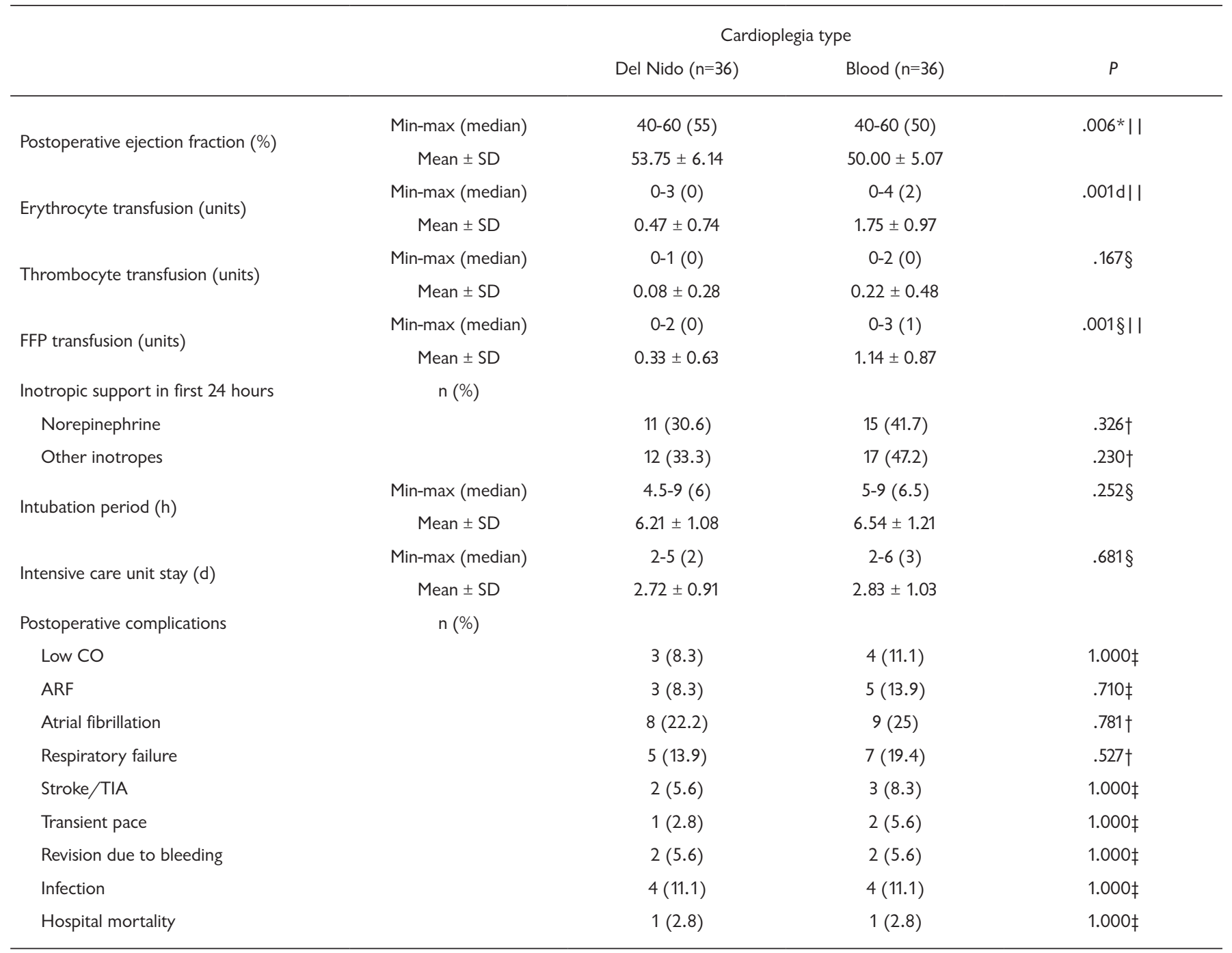

ARF indicates acute renal failure; FFP, fresh frozen plasma; *Student’s $t$ test; $\nmid$ Pearson $\chi^{2}$ test; $\ddagger$ Fisher’s exact test; $\S$ Mann-Whitney $U$ test; ||$P<.01$.

adding crystalloid-based solution in 1 part blood using a low cardioplegia volume. Hematocrit levels at hours 6 and 24 were significantly higher in the DNC group than in the BC group. DNC resulted in less use of erythrocyte transfusions than BC.

Additional benefits have been reported for DNC. Muktad et al [2018] evaluated micro-embolic events with a single dose of DNC and conventional multidose blood cardioplegia with transcranial Doppler ultrasonography. The use of a single dose of DNC was found to be associated with a 7 -fold decrease in the number of micro-emboli per minute of bypass.

There are some concerns related to DNC. In coronary artery disease, a uniform distribution is not guaranteed owing to the presence of large vessel disease and impaired microcirculation [Kim 2014]. The high potassium content of DNC is another issue, in that residual potassium can lead to coronary vasoconstriction and will complicate myocardial ischemia. DNC also has a low nutrient content of $21 \mathrm{kcal} / \mathrm{L}$. An energydepleted myocardium will be affected by low energy levels. Another concern is that multidosing with BC will also beneficially wash out toxic metabolites, which is not the case for DNC [Valooran 2016]. Data on redosing after 60 to 90 minutes and on the use of DNC during long-lasting complex cardiac surgery are lacking [Gorgy 2019]. Most previous studies were conducted in selected groups, and data on the use of DNC in multiple comorbid conditions or revision surgeries are lacking.

This study has some limitations. The retrospective nature of the design is the first limitation. The study group had a relatively small sample size; however, aortic root surgery is a relatively rare surgery. Short-term outcomes were presented in this study; however, data in the literature also noted that the short-term potential benefits did not affect the long-term outcomes [An 2019]. 


\section{Conclusion}

Del Nido cardioplegia offers some advantages in terms of a short cross-clamp time and a low cardioplegia volume. With the use of DNC, less defibrillation occurs. The related low levels of CK-MB are signs of better myocardial protection. However, data on the use of DNC in complex cardiac procedures and prospective randomized trials are lacking to establish its use in cardiac surgery. Our study extends previous observations of DNC to different cardiac procedures for aortic root surgeries.

\section{REFERENCES}

Ad N, Holmes SD, Massimiano PS, et al. 2018. The use of del Nido cardioplegia in adult cardiac surgery: A prospective randomized trial. J Thorac Cardiovasc Surg 155:1011-1018.

Ali JM, Miles LF, Abu-Omar Y, Galhardo C, Falter F. 2018. Global cardioplegia practices: Results from the Global Cardiopulmonary Bypass Survey. J Extra Corpor Technol ;50:83-93.

An KR, Rahman IA, Tam DY, et al. 2019. A systematic review and metaanalysis of del Nido versus conventional cardioplegia in adult cardiac surgery. Innovations (Phila) 14:385-393.

Doenst T, Borger MA, Weisel RD, et al. 2008. Relation between aortic cross-clamp time and mortality — not as straightforward as expected. Eur J Cardiothorac Surg 33:660-665.

Gorgy A, Shore-Lesserson L. 2019. Del Nido cardioplegia should be used in all adults undergoing cardiac surgery: Con. J Cardiothorac Vasc Anesth 33:1791-1794.

Kim K, Ball C, Grady P, Mick S. 2014. Use of del Nido cardioplegia for adult cardiac surgery at the Cleveland Clinic: Perfusion implications. J Extra Corpor Technol 46:317-323.

Kim WK, Kim HR, Kim JB, et al. 2018. del Nido cardioplegia in adult cardiac surgery: Beyond single-valve surgery. Interact Cardiovasc Thorac Surg 27:81-87.

Li Y, Lin H, Zhao Y, et al. 2018. Del Nido cardioplegia for myocardial protection in adult cardiac surgery: A systematic review and meta-analysis. ASAIO J 64:360-367.

Mongero LB, Tesdahl EA, Stammers AH, Stasko AJ, Weinstein S. 2018. Does the type of cardioplegia solution affect intraoperative glucose levels? A propensity-matched analysis. J Extra Corpor Technol 50:44-52.

Mukdad L, Toppen W, Sanaiha Y, et al. 2018. Incidence of cerebral microemboli in single-dose vs. multidose cardioplegia in adult cardiac surgery. J Extra Corpor Technol 50:143-148.

Nardi P, Pisano C, Bertoldo F, Ruvolo G. 2018. New insights on the use of del Nido cardioplegia in the adult cardiac surgery. J Thorac Dis 10(suppl 26):S3233-S3236.

Ota T, Yerebakan H, Neely RC, et al. 2016. Short-term outcomes in adult cardiac surgery in the use of del Nido cardioplegia solution. Perfusion 31:27-33.

Rosenbloom M, Hancock M, Weinstock P, et al. 2018. Del Nido cardioplegia for an aortic valve replacement patient with cold agglutinins. J Extra Corpor Technol 50(3):187-188.

Salis S, Mazzanti VV, Merli G et al. 2008. Cardiopulmonary bypass duration is an independent predictor of morbidity and mortality after cardiac surgery. J Cardiothorac Vasc Anesth 22:814-822.

Sorabella RA, Akashi H, Yerebakan H, et al. 2014. Myocardial protection using del nido cardioplegia solution in adult reoperative aortic valve surgery. J Card Surg 29:445-449.

Valooran GJ, Nair SK, Chandrasekharan K, Simon R, Dominic C. 2016. del Nido cardioplegia in adult cardiac surgery-scopes and concerns. Perfusion 31:6-14.

Vistarini N, Laliberté E, Beauchamp P, et al. 2017. Del Nido cardioplegia in the setting of minimally invasive aortic valve surgery. Perfusion 32:112-117. 